\title{
Information Entropy and Protein Secondary Structure in the ZEBOV- Makona Ebola Virus Glycoprotein
} Joel K Weltman*

Alpert Medical School, Brown University, Providence, RI 02912 USA

\begin{abstract}
The current epidemic of Ebola virus disease (EVD) is caused by Zaire Ebola virus-Makona variant. Results are presented indicating that $88 \%$ of the information entropy $(\mathrm{H})$ in the ZEBOV-Makona glycoprotein $(\mathrm{GP} 1,2)$ was distributed to amino acids residing in random coil structures. In contrast, only $12 \%$ of the total $\mathrm{H}$ was due to mutations of amino acids in helical and extended sheet secondary structures. It is proposed that some of the $\mathrm{H}$ in random coils may represent mutational escape from host defense while the paucity of $\mathrm{H}$ in helical and extended sheet structures may reflect conformational constraints on mutation. By relating GP1,2 secondary structure and $\mathrm{H}$ in regions of GP1,2, this research helps to computationally identify potential targets for the design of anti-Ebola vaccines and drugs.
\end{abstract}

Keywords: Zaire Ebola virus; ZEBOV-Makona variant; Information entropy; Glycoprotein; GP; GP1,2; Protein secondary structure; EVD; Vaccines

\section{Introduction}

The high fatality of the 2014-2015 epidemic of Ebola virus disease (EVD) has been caused by the Zaire Ebola virus-Makona variant (ZEBOV-Makona) [1]. The research reported here focuses on distributions of information entropy $(\mathbf{H})$ [2] and secondary structure [3] in the ZEBOV-Makona glycoprotein $(\mathbf{G P 1 , 2})$. The GP1,2 is the viral protein that mediates the binding and internalization of the virus by the target cell $[4,5]$.

The high mortality, morbidity and public-health significance of EVD stresses the importance of developing effective vaccines, treatments and point-of-care diagnostics for this disease [6]. Results are presented that indicate most of the total $\mathbf{H}$ distribution in the GP1,2 protein is statistically accounted for by amino acids participating in random coil secondary structures. Knowledge of the $\mathbf{H}$ distribution in ZEBOV-Makona GP1,2, and knowledge of the structural features of that distribution, increase our insight into the functional biology of this virus. By helping to identify appropriate potential targets, this insight can facilitate the design of preventive and therapeutic anti-Ebola vaccines and drugs.

\section{Materials and Methods}

The complete set $(\mathrm{N}=877)$ of Zaire Ebola virus ZEBOV-Makona variant GP1,2 full-length nucleotide sequences (length $=2031$ nucleotides) was downloaded in FASTA format [7] on September 3, 2015 using the NCBI Ebolavirus Resource (http://www.ncbi.nlm.nih. gov/genome/viruses/variation/ebola/). The downloaded set of 877 GP1,2 nucleotide gene sequences was translated into amino acids with Biopython 1.65, using the IUPAC unambiguous DNA code. Eight hundred and fifteen (815) of these translated GP1,2 protein sequences were of full length 676 amino acids and without error characters. These 815 full-length, error-free GP1,2 protein sequences, comprising 92.93\% of the initial download of nucleotide sequenes, were utilized without further sequence re-alignment.

Information entropy $(\mathbf{H})$ was calculated by the equation of Shannon [8]. Computation and graphing were performed with 64-bit Enthought Canopy 1.5.1, Python 2.7.6, Numpy 1.9.2-1, Scipy 0.15.1-2 and matplotlib 1.4.2-2. Mann-Whitney U tests were computed with the SciPy stats module; two-tail $\mathrm{p}$ values are reported.
The consensus sequence of the GP1,2 dataset was determined with Jalview (2.8.2) [9]. Scores for random coil, helix and extended sheet predicted secondary structures were obtained for the consensus sequence with the PSIPRED Protein Structure Prediction Server [10]. For representation of a variation of $\mathbf{H}$ distribution dependent upon protein secondary structure, each value of $\mathbf{H}$ was assigned to the mathematical array representing the secondary structure reported by PsiPred.

\section{Results and Discussion}

PsiPred scores summed along the length of the GP1,2 sequence are shown in Figure 1 for random coil, helical and extended sheet secondary structures. The summed random coil scores were significantly greater than the summed scores for helices $(U=23297.0, p=3.2326 \mathrm{e}-182)$ and the summed scores for extended sheets $(U=49522.0, p=7.8439 \mathrm{e}-138)$. The summed scores for helices were larger than those for extended sheets (127759.0, $\mathrm{p}=1.5488 \mathrm{e}-45)$. The total, summed Psipred score was 380.959 for random coil, 95.239 for helix and 53.068 for extended sheets. Counting of PsiPred output showed that total number of amino acids in random coils $(\mathrm{N}=485)$ was greater than the number either helices $(\mathrm{N}=111)$ or in extended sheets $(\mathrm{N}=80)$. The results in Figure 1 show that the predominant predicted secondary structure of GP1,2 is the random coil. The predicted distribution of each of three secondary structures along the length of the GP1,2 protein is shown in Figure 2. Mann-Whitney $U$ tests on these unsummed distributions show that random coil scores were significantly greater than those for helices $(\mathrm{U}=111216.0, \mathrm{p}=2.5389 \mathrm{e}-72)$ and for extended sheets $(\mathrm{U}=83790.0$, $\mathrm{p}=4.2439 \mathrm{e}-112)$. The PsiPred scores for helices were slightly greater than those for extended sheets $(U=215249.5, p=0.0023)$. Thus, the statistically ranked PsiPred scores for GP1,2 secondary structures are: random coils $>$ helices $>$ extended sheet. Plots of the PsiPred score

*Corresponding author: Joel K. Weltman, Clinical Professor Emeritus of Medicine, Alpert Medical School, Brown University, Providence, RI 02912 USA Tel: 401-863-1000; E-mail: joel_weltman@brown.edu

Received October 08, 2015; Accepted November 09, 2015; Published November 16,2015

Citation: Weltman JK (2015) Information Entropy and Protein Secondary Structure in the ZEBOV-Makona Ebola Virus Glycoprotein. J Health Med Informat 6: 207. doi:10.4172/2157-7420.1000207

Copyright: (c) 2015 Weltman JK. This is an open-access article distributed under the terms of the Creative Commons Attribution License, which permits unrestricted use, distribution, and reproduction in any medium, provided the original author and source are credited. 


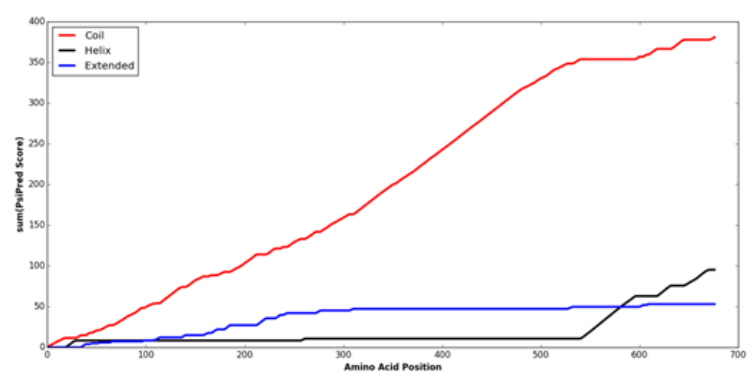

Figure 1: Summed PsiPred Scores for GP1,2 Secondary Structures.
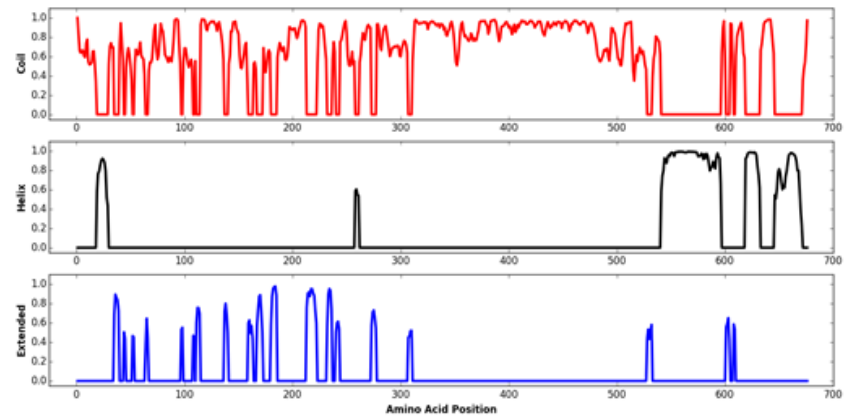

Figure 2: Predicted Secondary Structure of ZEBOV-Makona Glyoprotein. (top) random coil (middle) helix (bottom) extended sheet. $x=a m i n o$ acid position, $y=$ PsiPRED score for indicated protein secondary structure.

distributions in Figure 2 show that the random coil distribution was most uniformly dominant over the distributions of the other two structures between positions 311-527. Helices were located near the termini, especially the C-terminus. Regions with extended sheets were dispersed primarily in the first half of the GP1,2 protein, ie, between amino acid positions 35 and 310 .

The GP1,2 amino acid $\mathbf{H}$ values were sorted into three sets, depending upon the secondary structure predicted for each of the 676 amino acid positions. Summation curves of $\mathbf{H}$ as a function of amino acid position are shown in Figure 3 for the three structure-based sets, The summation curve for the random coil set differed both from the curve for the helix set $(U=53030.5, p=1.2230 \mathrm{e}-139)$ and for the extended sheet set $(\mathrm{U}=42201 . \mathrm{p}=1.2461 \mathrm{e}-151)$. However, the summation curve for the helix set differed only slightly from that of the extended sheet set ( $U=207878.5, p=0.0028)$. The total summed $\mathbf{H}$ for the random coil set (4.3742 bits) was greater than the total summed $\mathbf{H}$ for either the helix set $(0.3616$ bits $)$ or for the set of amino acids in extended sheets $(0.2351$ bits). Thus, $88.00 \%$ of the information entropy $(\mathbf{H})$ in the ZEBOVMakona glycoprotein (GP1,2) was in random coil structures, $7.27 \%$ in helices and $4.73 \%$ in extended sheets.

Distributions of $\mathbf{H}$ in the secondary structures of the ZEBOVMakona glycoprotein without summation are shown in Figure 4. It is clearly seen that although some $\mathbf{H}$ was observed in helices and extended sheets, most of the total $\mathbf{H}$ distribution in the GP1,2 protein is accounted for by amino acids participating in random coil secondary structures. $\mathbf{U}$-tests on these data showed that the $\mathbf{H}$ values in random coils were significantly greater than the $\mathbf{H}$ values in either helices $(U=203115.5, p=7.8279 \mathrm{e}-16)$ or extended sheets $(U=203614.5$, $\mathrm{p}=5.2477 \mathrm{e}-15)$. In contrast, $\mathbf{H}$ distributions in the helical and random sheet structures were not statistically distinguishable from each other $(U=227826.5, p=0.6597)$. It should be noted that the statistical predominance of random coil $\mathbf{H}$ values over the $\mathbf{H}$ values of helices and sheets does not merely reflect the greater total number of positions with random coil structure; the statistical predominance of random coil $\mathbf{H}$ is also a reflection of the propensity of individual GP1,2 amino acid positions with random coil structure to be linked to the greater observed $\mathbf{H}$ values per position. For example, the GP1,2 amino acid positions with highest ranking $\mathbf{H}$ values in the three sets (coil, helix, extended sheet) were positions 82, 230 and 371. As shown in Figure 4 , these amino acids all were in random coil structures. These three amino acid positions recently have been shown to be associated with disjoint, but complete, cliques of amino acids networked by mutual information within the GP1,2 molecule [11]. Thus, the observed mutational distributions, expressed as $\mathbf{H}$ distributions, are linked to Ebola virus function and evolutionary trajectory.

The amino acids where $\mathbf{H}>0$ between positions 54-201 lie within the GP1,2 receptor binding domain (RBD) $[12,13]$ and thus, the positive $\mathbf{H}$ values in this region may represent viral evasive mutations in response to defensive immunologic and other biological forces of the host $[14,15]$. Epitopic peptide sequences where $\mathbf{H}=0$ were reported to exist between mutating sites within the RBD of Zaire and Sudan strains of Ebola virus [16,17]; these sequences are also found within the RBD of the Makona variant reported here. These RBD peptides are describable predominantly as random coil structure and thus, should be effective carriers and displayers of Ebola glycoprotein linear epitopes. Two of the peptides where $\mathbf{H}=0$ at all positions are the $24 \mathrm{mer}$ (positions 83-106):

\section{TKRWGFRSGVPPKVVNYEAGEWAE}

and the 17mer (positions 112-128):

\section{EIKKPDGSECLPAAPDG.}

These two peptides together are comprised of 41 amino acids, of which $5(12.20 \%)$ are in extended sheets; the remaining 38 residues $(87.80 \%)$ are in random coils.

In contrast to the high entropy positions in the RBD, discussed above, there were 32 low entropy, extended sheet structures within the ZEBOV-Makona RBD. These low entropy positions may reflect mutational constraints that are conformationally imposed. Extended sheets are concentrated in the RBD of the glycoprotein (Figure 2, bottom). The low values of $\mathbf{H}$ in the extended sheets are consistent with an increase in inter-amino acid side chain interactions that physically stabilize the sheet structure. Such physical stabilization would make emergence of successful mutations in the sheets less probable. Unlike the helix and extended sheet structures with intramolecular conformational constraints, random coil structures tend to be open and solvent accessible [18]. Thus, stabilized extended sheet amino acids, surrounded by penetrable coil structures, could be attractive targets for anti-EBOLA antibodies, especially if the sheets are located in the RBD which is essential for viral function.

\section{Conclusions}

The presented results show that information entropy in the ZEBOV-Makona glycoprotein is distributed mainly to amino acid positions in random scoil structures. It is proposed that the random coil glycoprotein regions with $\mathbf{H}>\mathbf{0}$ represent sites of interaction of Ebola virus with its external environment, resulting in evolution towards mutational escape. The presented results also suggest that the observed low incidence of mutations in extended sheet positions may 
Citation: Weltman JK (2015) Information Entropy and Protein Secondary Structure in the ZEBOV-Makona Ebola Virus Glycoprotein. J Health Med Informat 6: 207. doi:10.4172/2157-7420.1000207

Page 3 of 3

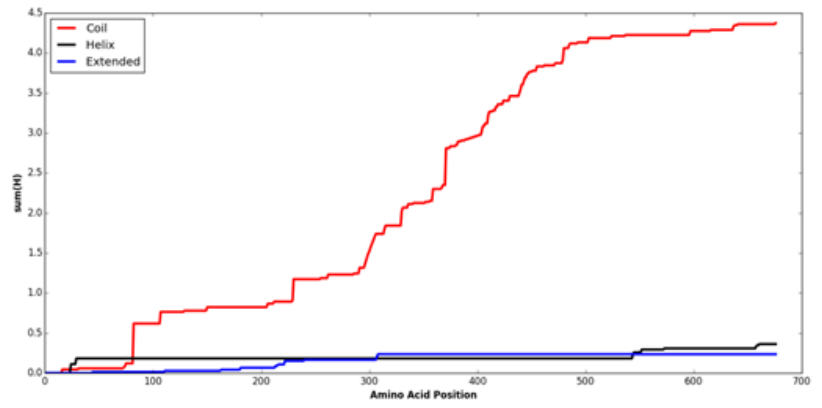

Figure 3: Summed Distributions of Information Entropy in Secondary Structures of ZEBOV-Makona Glycoprotein. $x=a m i n o$ acid position; $y=$ summed information entropy $(\operatorname{sum}(\mathrm{H}))$, in bits.

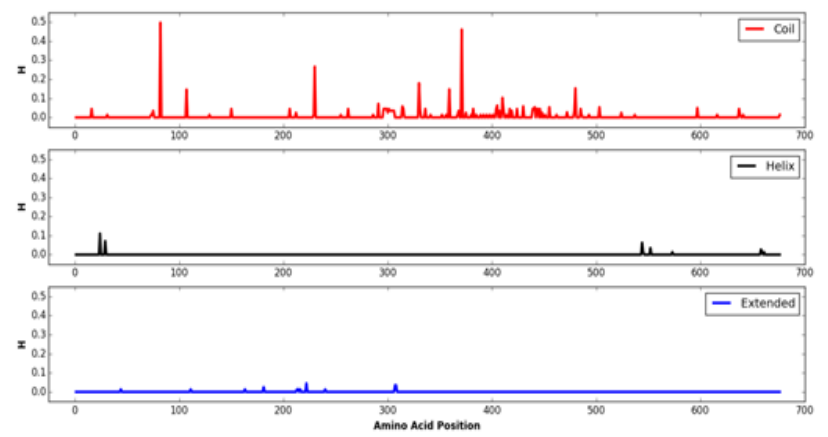

Figure 4: Information Entropy Distributions in Secondary Structures of ZEBOV-Makona Glycoprotein. (top) random coil positions (middle) helical positions (bottom) extended sheets. $x=a m i n o$ acid position, $y=$ information entropy $(\mathrm{H})$, in bits.

be caused by conformational constraints. Conformational epitopes displayed by the sheets, especially within the RBD, would be attractive targets for development of ant-Ebola vaccines and immunological therapies. Thus, this research helps provide insight for the development of vaccines against both linear and conformational Ebola epitopes.

\section{References}

1. Kuhn JH, Andersen KG, Baize S, Bào Y, Bavari S, et al (2014) Nomenclature and Database-Compatible Names for the Two Ebola Virus Variants that Emerged in Guinea and the Democratic Republic of the Congo in 2014. Viruses 6: 4760-4799.
2. Cover TM and Thomas JA (2006) Entropy, Relative Entropy and Mutual Information. In Elements of Information Theory. (2ndedn) Wiley, USA.

3. Kabsch W, Sander C (1983) Dictionary of protein secondary structure: pattern recognition of hydrogen-bonded and geometrical features. Biopolymers 22 2577-2637.

4. Lee JE and Saphire EO (2009) Ebolavirus glycoprotein structure and mechanism of entry. Future Virol 6: 621-635.

5. Miller EH, Chandran K (2012) Filovirus entry into cells - new insights. Curr Opin Virol 2: 206-214.

6. Kaushik A, Tiwari S, Jayant RD, Marty A, Nair M (2016) Towards detection and diagnosis of Ebola virus disease at point-of-care. Biosens Bioelectron 75 : 254-272.

7. 7. Lipman DJ, Pearson WR (1985) Rapid and sensitive protein similarity searches. Science 227: 1435-1441.

8. Shannon, Claude E (1948) A Mathematical Theory of Communication. Bell System Technical Journal 27: 379-423.

9. Waterhouse AM, Procter JB, Martin DM, Clamp M, Barton GJ (2009) Jalview Version 2--a multiple sequence alignment editor and analysis workbench. Bioinformatics 25:1189-1191.

10. Buchan DWA, Minneci F, Nugent TCO, Bryson K, Jones DT (2013) Scalable web services for the PSIPRED Protein Analysis Workbench. Nucleic Acids Res 41: W340-W348.

11. Weltman JK (2015) Mutual Information-Based Cliques of Amino Acids in the Zaire Ebola Virus-Makona Glycoprotein; In Proceedings of the 2nd Int. Electron Conf. Entropy Appl. in the press.

12. Dube D, Brecher MB, Delos SE, Rose SC, Park EW, et al. (2009) The primed ebolavirus glycoprotein (19-kilodalton GP2): sequence and residues critical for host cell binding. J Virol 83: 2883-2891.

13. Lee JE, Fusco ML, Hessell AJ, Oswald WB, Burton DR, et al. (2008) Structure of the Ebola virus glycoprotein bound to an antibody from a human survivor. Nature 454: 177-182.

14. Vossen MT, Westerhout EM, Söderberg-Nauclér C and Wiertz EJ (2002)Vira immune evasion: a masterpiece of evolution. Immunogenetics. 54:527-542.

15. De Groot AS, Knopf PM, Rivera D, Martin W (2008) Immunoinformatics Applied to Modifying and Improving Biological Therapeutics; in Immunoinformatics; C Schönbach, S Ranganathan and V Brusic Springer : 109-131.

16. Weltman JK (2014) Identification of Invariant Peptide Domains within Ebola Virus Glycoprotein GP1,2. J Med Microb Diagn 4: 176

17. Weltman JK (2014) Combined Use of Information Entropy and Bepipred Scores for Screening Ebola Virus Glycoprotein (GP) Sequences. In Proceedings of the 1st Int. Electron Conf. Entropy Appl. Sciforum Electronic Conference Series 1: d003.

18. Lins L, Thomas A and Brasseur R (2003) Analysis of accessible surface of residues in proteins. Protein Sci 12: 1406-1417. 